\title{
A Fringe Center Detection Technique Based on a Sub-Pixel Resolution, and Its Applications Using Sinusoidal Gratings
}

\author{
Ming Chang \\ Chung Yuan Christian University \\ Paul P. Lin \\ Cleveland State University, p.lin@csuohio.edu \\ Wen Chih Tai \\ Chung Yuan Christian University \\ Follow this and additional works at: https://engagedscholarship.csuohio.edu/enme_facpub \\ Part of the Computer-Aided Engineering and Design Commons, and the Optics Commons \\ How does access to this work benefit you? Let us know!
}

\section{Publisher's Statement}

NOTICE: this is the author's version of a work that was accepted for publication in Optics and Lasers in Engineering. Changes resulting from the publishing process, such as peer review, editing, corrections, structural formatting, and other quality control mechanisms may not be reflected in this document. Changes may have been made to this work since it was submitted for publication. A definitive version was subsequently published in Optics and Lasers in Engineering, 20, 3, (01-01-1994); 10.1016/S0143-8166(94)90011-6

\section{Original Citation}

Chang, M., Lin, P. P., , \& Tai, W. C. (1994). A fringe center detection technique based on a sub-pixel resolution, and its applications using sinusoidal gratings. Optics and Lasers in Engineering, 20(3), 163-176. doi:10.1016/S0143-8166(94)90011-6

This Article is brought to you for free and open access by the Mechanical Engineering Department at EngagedScholarship@CSU. It has been accepted for inclusion in Mechanical Engineering Faculty Publications by an authorized administrator of EngagedScholarship@CSU. For more information, please contact library.es@csuohio.edu. 


\title{
A Fringe Center Detection Technique Based on a Sub-pixel Resolution, and its Applications Using Sinusoidal Gratings
}

\author{
Ming Chang, ${ }^{a}$ Paul P. $\operatorname{Lin}^{b *} \&$ Wen Chih Tai ${ }^{a}$ \\ aDepartment of Mechanical Engineering, Chung Yuan Christian University, Chung Li, \\ Taiwan \\ ${ }^{h}$ Mechanical Engineering Department, Fenn College of Engineering, Cleveland State \\ University, Cleveland, Ohio 44115, USA
}

\begin{abstract}
A common problem in optical profilometry is the accuracy in locating fringe centers. This paper presents an accurate fringe center detection technique based on sub-pixel resolution using the fringe projection method. An optimum reconstruction filter is developed which has low sensitivity to noise. In fringe center detection, computer simulation results of using one-pixel and sub-pixel resolutions are compared. The detection technique is then applied to radius measurement of cylindrical objects and surface profile measurement of diffuse objects. The experimental results thus obtained through the proposed optimum reconstruction filter show significant improvement in measurement accuracy.
\end{abstract}

\section{INTRODUCTION}

The fringe projection method, ${ }^{1-3}$ is a well-known technique for noncontact profilometric measurement of diffuse objects. In using this method, optical fringes are generated by projecting a grating onto an object. These fringes are deformed owing to the variation in geometry of the object. The simplest profilometric algorithm is to detect the center location of each deformed fringe and calculate the curvatures of the detected fringe centers so that the third dimension (perpendicular to the camera coordinates) of the object geometry can be determined.

Lin et $a l{ }^{4}$ developed an accurate fringe center detection technique based on sub-pixel resolution. They projected a moiré grating (also

* To whom correspondence should be addressed. 
called Ronchi ruling) onto an object surface to generate fringes. It was found that the intensity distribution across a fringe resembled a Gaussian distribution. In this research, a grating of sinusoidal intensity distribution is used so that the surface height distribution can be translated into a phase distribution, and the method of phase modulation interferometry can be applied for a quantitative analysis of surface topography. Recent developments in interferometry-based profilometry include a new phase reduction algorithm by Wan and Lin, ${ }^{5}$ a phase mapping approach in phase-measuring profilometry by Srinivasan et $a l .{ }^{3}$ and on-line automatic phase-measuring profilometry by Chang and Wan. ${ }^{6}$

This paper shows how to detect the fringe centers approximately, and then locate these fringes more accurately on the basis of sub-pixel resolution. An optimum reconstruction filter is developed which has low sensitivity to noise. Computer simulation and experimental results are also presented.

\section{FRINGE CENTER DETECTION BASED ON 1-PIXEL RESOLUTION}

Figure 1 is a schematic diagram of a measuring system using the fringe projection method. The system consists of a sinusoidal grating, the grating projector, a charge-coupled device (CCD) camera, an image processor, and a computer. The intensity distribution of the deformed fringes is recorded by the CCD camera and digitally transmitted to the computer through the frame grabber. A low-pass filter is used to reduce noise. The fringe width and curvature may vary from place to place depending upon the object's geometry. The fringe curvature can be

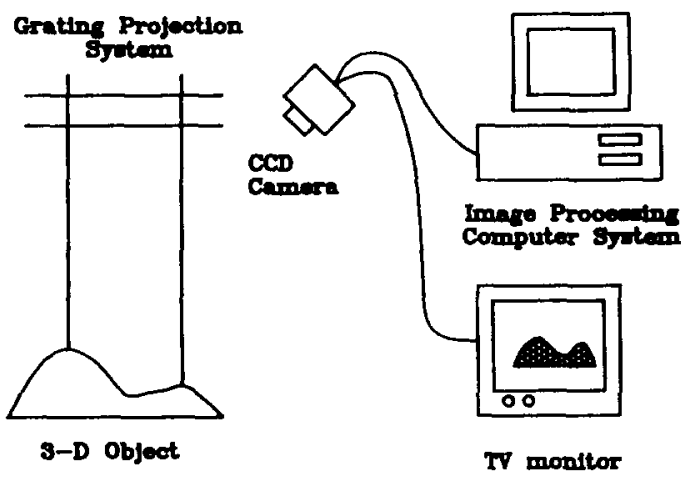

Fig. 1. Schematic diagram of the fringe projection system. 
calculated by detecting the fringe centers along a fringe row by row and connecting them from top to bottom. The fringe center should be located at the peak of an intensity distribution curve. The easiest way to locate the fringe centers approximately is to find the column positions where the highest and lowest pixel gray level values exist per row per fringe. An efficient algorithm based on a 1-pixel resolution to locate the intensity extremes is stated as follows:

the intensity maximum exists at column $m$

$$
\text { if } I_{m}>I_{m+k}+J \text { and } I_{m}>I_{m-k}+J \text { are true }
$$

the intensity minimum exists at column $n$

$$
\text { if } I_{n}<I_{n+k}-J \text { and } I_{n}<I_{n-k}-J \text { are true }
$$

where $J$ stands for a threshold value and $k$ is a constant depending upon the fringe width. The algorithm works more efficiently when $k$ is about three, but no less than three. The accuracy of fringe center detection based on 1-pixel resolution (as shown in column 1 of Tables 1 and 2 when $k=3$ ) is poor, although the computational efficiency is great.

\section{FRINGE CENTER DETECTION BASED ON SUB-PIXEL RESOLUTION}

When a sinusoidal grating is projected onto an object, the detected intensity distribution across a scanned line still resembles a sinusoidal function, except that it slightly varies with conditions such as illumination, focus/blur, and noise. The light intensity function can be expressed as

$$
I(x)=A \times \sin (\alpha x+\beta)+B
$$

where $I(x)$ is the pixel intensity at column $x$, and $\alpha, \beta, A$, and $B$, are the wavenumber, initial phase, amplitude, and the minimum gray level of the periodical distribution, respectively.

\section{Theoretical estimate of fringe center detection error}

Theoretically, the exact fringe center can be found by differentiating $I(x)$ with respect to $x$ and calculating $x$ from $I^{\prime}(x)=0$. Thus, the exact center is located at $x_{\mathrm{c}}=[(\pi / 2)-\beta] / \alpha$. As

$$
\begin{aligned}
I(x) & =A \sin (\alpha x+\beta)+B \\
& =A(\sin \alpha x \cos \beta+\cos \alpha x \sin \beta)+B
\end{aligned}
$$

the intensity function can be approximated by a Taylor's series expansion about $x_{\mathrm{c}}$ to second order. Thus,

$$
I(x) \approx c_{2} x^{2}+c_{1} x+c_{0}
$$


TABLE 1

Computer Simulation of Fringe Center Detection without Random Noise (Values Given in Pixels)

\begin{tabular}{|c|c|c|c|c|c|c|c|}
\hline \multirow[t]{2}{*}{ Wavelength } & \multirow{2}{*}{$\frac{\begin{array}{c}\text { One-pixel } \\
\text { resolution }\end{array}}{\text { Eqn (1) }}$} & \multicolumn{6}{|c|}{ Sub-pixel resolution } \\
\hline & & Lagrange & Cubic & Least squares & Gaussian & B-Spline & Bernstein \\
\hline \multicolumn{8}{|c|}{ (a) Using 3 pixels: $x_{-1}, x_{0}, x_{1}$} \\
\hline 55.0000 & 0.3928 & $0 \cdot 1426$ & $0 \cdot 1511$ & $0 \cdot 1426$ & $0 \cdot 3917$ & $0 \cdot 3173$ & $0 \cdot 3173$ \\
\hline $27 \cdot 5000$ & $0 \cdot 2822$ & $0 \cdot 0415$ & $0 \cdot 0897$ & 0.0415 & $0 \cdot 3690$ & $0 \cdot 2942$ & $0 \cdot 2942$ \\
\hline $18 \cdot 3333$ & $0 \cdot 2671$ & 0.0234 & 0.0948 & 0.0234 & $0 \cdot 2927$ & 0.2511 & $0 \cdot 2511$ \\
\hline $13 \cdot 7500$ & $0 \cdot 2712$ & $0 \cdot 0121$ & $0 \cdot 0906$ & $0 \cdot 0121$ & $0 \cdot 2904$ & $0 \cdot 2516$ & $0 \cdot 2516$ \\
\hline $11 \cdot 0000$ & 0.2602 & $0 \cdot 0120$ & $0 \cdot 1047$ & 0.0122 & $0 \cdot 2733$ & 0.2341 & $0 \cdot 2341$ \\
\hline $9 \cdot 1667$ & $0 \cdot 2640$ & 0.0109 & 0.0939 & $0 \cdot 0109$ & $0 \cdot 2316$ & 0.2427 & $0 \cdot 2427$ \\
\hline \multicolumn{8}{|c|}{ (b) Using 5 pixels: $x_{-2}, x_{-1}, x_{0}, x_{1}, x_{2}$} \\
\hline $55 \cdot 0000$ & 0.3928 & $0 \cdot 3561$ & $0 \cdot 3470$ & $0 \cdot 1052$ & $0 \cdot 3854$ & $0 \cdot 2306$ & $0 \cdot 2306$ \\
\hline $27 \cdot 5000$ & $0 \cdot 2822$ & $0 \cdot 0505$ & 0.0709 & 0.0175 & $0 \cdot 2650$ & 0.0976 & 0.0976 \\
\hline $18 \cdot 3333$ & 0.2671 & $0 \cdot 0260$ & 0.0337 & 0.0099 & 0.2326 & 0.0864 & $0 \cdot 0864$ \\
\hline $13 \cdot 7500$ & $0 \cdot 2712$ & $0 \cdot 0126$ & $0 \cdot 0207$ & $0 \cdot 0088$ & $0 \cdot 2113$ & $0 \cdot 0851$ & $0 \cdot 0851$ \\
\hline $11 \cdot 0000$ & $0 \cdot 2602$ & $0 \cdot 0079$ & $0 \cdot 0132$ & $0 \cdot 0170$ & $0 \cdot 1725$ & $0 \cdot 0770$ & $0 \cdot 0770$ \\
\hline $9 \cdot 1667$ & $0 \cdot 2640$ & 0.0059 & $0 \cdot 0086$ & $0 \cdot 0212$ & $0 \cdot 1446$ & 0.0767 & $0 \cdot 0767$ \\
\hline \multicolumn{8}{|c|}{ (c) Using 7 pixels: $x_{-3}, x_{2}, x_{-1}, x_{0}, x_{1}, x_{2}, x_{3}$} \\
\hline $55 \cdot 0000$ & 0.3928 & 0.3466 & $0 \cdot 3380$ & $0 \cdot 0412$ & $0 \cdot 3864$ & $0 \cdot 1055$ & $0 \cdot 1055$ \\
\hline $27 \cdot 5000$ & $0 \cdot 2822$ & $0 \cdot 0535$ & $0 \cdot 0623$ & 0.0091 & $0 \cdot 2650$ & $0 \cdot 0563$ & $0 \cdot 0563$ \\
\hline $18 \cdot 3333$ & $0 \cdot 2671$ & $0 \cdot 0281$ & $0 \cdot 0326$ & $0 \cdot 0100$ & 0.2326 & 0.0503 & 0.0503 \\
\hline $13 \cdot 7500$ & $0 \cdot 2712$ & $0 \cdot 0147$ & $0 \cdot 0178$ & 0.0186 & $0 \cdot 2113$ & $0 \cdot 0512$ & $0 \cdot 0512$ \\
\hline $11 \cdot 0000$ & $0 \cdot 2602$ & $0 \cdot 0091$ & $0 \cdot 0127$ & 0.0293 & $0 \cdot 1725$ & 0.0449 & 0.0449 \\
\hline $9 \cdot 1667$ & $0 \cdot 2640$ & 0.0063 & 0.0074 & $0 \cdot 0447$ & $0 \cdot 1446$ & $0 \cdot 0414$ & 0.0414 \\
\hline
\end{tabular}

Gray level value $=100+100 \cos (2 \pi z /$ wavelength $)+$ random $(0)$

$z=r-\left(r^{2}-y^{2}\right)^{1 / 2}+E y+F x$, where $E=0, F=1$.

Note: random $(0)$ : no random noise added to the gray value. 
TABLE 2

Computer Simulation Results with Random Noise (Values Given in Pixels)

\begin{tabular}{|c|c|c|c|c|c|c|c|}
\hline \multirow[t]{2}{*}{ Wavelength } & \multirow{2}{*}{$\begin{array}{c}\begin{array}{c}\text { One-pixel } \\
\text { resolution }\end{array} \\
\text { Eqn }(I)\end{array}$} & \multicolumn{6}{|c|}{ Sub-pixel resolution } \\
\hline & & Lagrange & Cubic & Least squares & Gaussian & B-Spline & Bernstein \\
\hline \multicolumn{8}{|c|}{ (a) Using 3 pixels: $x_{-1}, x_{0}, x_{1}$} \\
\hline $55 \cdot 0000$ & $0 \cdot 3787$ & $0 \cdot 3023$ & $0 \cdot 3886$ & $0 \cdot 3023$ & $0 \cdot 4977$ & $0 \cdot 6192$ & $0 \cdot 6192$ \\
\hline $27 \cdot 5000$ & $0 \cdot 2930$ & $0 \cdot 2140$ & $0 \cdot 1863$ & $0 \cdot 2139$ & $0 \cdot 2617$ & $0 \cdot 3668$ & $0 \cdot 3668$ \\
\hline $18 \cdot 3333$ & $0 \cdot 2727$ & $0 \cdot 0676$ & $0 \cdot 1092$ & $0 \cdot 0677$ & $0 \cdot 4097$ & $0 \cdot 2688$ & $0 \cdot 2688$ \\
\hline $13 \cdot 7500$ & $0 \cdot 2704$ & $0 \cdot 0388$ & 0.0922 & $0 \cdot 0389$ & $0 \cdot 3957$ & $0 \cdot 2568$ & 0.2568 \\
\hline $11 \cdot 0000$ & $0 \cdot 2602$ & $0 \cdot 0279$ & $0 \cdot 0920$ & $0 \cdot 0279$ & $0 \cdot 3571$ & $0 \cdot 2367$ & $0 \cdot 2367$ \\
\hline $9 \cdot 1667$ & $0 \cdot 2636$ & $0 \cdot 0202$ & $0 \cdot 0905$ & $0 \cdot 0201$ & $0 \cdot 2489$ & $0 \cdot 2412$ & $0 \cdot 2412$ \\
\hline \multicolumn{8}{|c|}{ (b) Using 5 pixels: $x_{-2}, x_{-1}, x_{0}, x_{1}, x_{2}$} \\
\hline $55 \cdot 0000$ & 0.3843 & $0 \cdot 6605$ & 0.6102 & $0 \cdot 2472$ & $0 \cdot 3800$ & $0 \cdot 4448$ & 0.4448 \\
\hline $27 \cdot 5000$ & 0.2926 & $0 \cdot 2186$ & $0 \cdot 2248$ & 0.0501 & 0.2766 & 0.1107 & $0 \cdot 1107$ \\
\hline $18 \cdot 3333$ & $0 \cdot 2677$ & $0 \cdot 0817$ & $0 \cdot 1009$ & $0 \cdot 0241$ & $0 \cdot 2336$ & 0.0916 & 0.0916 \\
\hline $13 \cdot 7500$ & 0.2712 & $0 \cdot 0443$ & 0.0539 & $0 \cdot 0154$ & $0 \cdot 2112$ & 0.0874 & 0.0875 \\
\hline $11 \cdot 0000$ & 0.2605 & $0 \cdot 0264$ & 0.0329 & 0.0179 & $0 \cdot 1737$ & 0.0785 & $0 \cdot 0784$ \\
\hline $9 \cdot 1667$ & $0 \cdot 2639$ & $0 \cdot 0193$ & $0 \cdot 0235$ & $0 \cdot 0228$ & $0 \cdot 1459$ & $0 \cdot 0755$ & $0 \cdot 0755$ \\
\hline \multicolumn{8}{|c|}{ (c) Using 7 pixels: $x_{-3}, x_{-2}, x_{-1}, x_{0}, x_{1}, x_{2}, x_{3}$} \\
\hline $55 \cdot 0000$ & $0 \cdot 3492$ & 0.6067 & 0.5612 & $0 \cdot 1258$ & $0 \cdot 3452$ & $0 \cdot 1832$ & $0 \cdot 1832$ \\
\hline $27 \cdot 5000$ & $0 \cdot 2938$ & $0 \cdot 2123$ & $0 \cdot 2245$ & $0 \cdot 0289$ & $0 \cdot 2779$ & $0 \cdot 0668$ & $0 \cdot 0667$ \\
\hline $18 \cdot 3333$ & $0 \cdot 2687$ & $0 \cdot 0845$ & $0 \cdot 0985$ & $0 \cdot 0167$ & $0 \cdot 2346$ & $0 \cdot 0554$ & $0 \cdot 0553$ \\
\hline $13 \cdot 7500$ & $0 \cdot 2710$ & $0 \cdot 0470$ & $0 \cdot 0545$ & $0 \cdot 0200$ & $0 \cdot 2112$ & $0 \cdot 0510$ & $0 \cdot 0510$ \\
\hline $11 \cdot 0000$ & $0 \cdot 2603$ & 0.0291 & $0 \cdot 0337$ & $0 \cdot 0298$ & $0 \cdot 1739$ & $0 \cdot 0470$ & $0 \cdot 0470$ \\
\hline $9 \cdot 1667$ & 0.2637 & 0.0204 & $0 \cdot 0238$ & $0 \cdot 0451$ & $0 \cdot 1453$ & 0.0422 & $0 \cdot 0422$ \\
\hline
\end{tabular}

Gray $=100+100 \cos (2 \pi z /$ wavelength $)+\operatorname{random}(3)$

$z=r\left(r^{2}-y^{2}\right)^{1 / 2}+E y+F x$, where $E=0, F=1$.

Note: random(3): random noise varying from zero to three gray values. 
where $\quad c_{2}=-A \alpha^{2} / 2, \quad c_{1}=A \alpha(\pi / 2-\beta) \quad$ and $\quad c_{0}=-A\left[(\pi / 2-\beta)^{2} /\right.$ $2-1]+B$.

To estimate the fringe detection error, it is assumed that $A=1$, $B=1, \alpha=0.5$ and $\beta=0.5$. The location of $X_{\mathrm{c}}$ is calculated at 2.14159 pixels. As the fringe center is approximately detected using 1-pixel resolution, the worst case will be when the detected center is 0.5 pixel away from the calculated center. For computational efficiency, a value $I(x)$ near the center is approximated by taking only five terms-two before the detected center and another two after the center. They are designated as $x_{-2}, x_{-1}, x_{0}, x_{1}$ and $x_{2}$, where $x_{0}$ is the location of the approximately detected center based on 1-pixel resolution. These five points are listed as follows:

$$
\begin{aligned}
x_{-2} & =0.64159 ; & I\left(x_{-2}\right) & =1.73169 \\
x_{-1} & =1.64159 ; & I\left(x_{-1}\right) & =1.96891 \\
x_{0} & =2.64159 ; & I\left(x_{0}\right) & =1.96891 \\
x_{1} & =3.64159 ; & I\left(x_{1}\right) & =1.73169 \\
x_{2} & =4.64159 ; & I\left(x_{2}\right) & =1.31532
\end{aligned}
$$

It should be noted that eqn (2) was used to calculate the theoretical $I(x)$ values. The above five points are then smoothed using a leastsquares second-order curve-fitting method. The estimated center is located at 2.15664 which is the peak of the fitted curve. Figure 2 shows

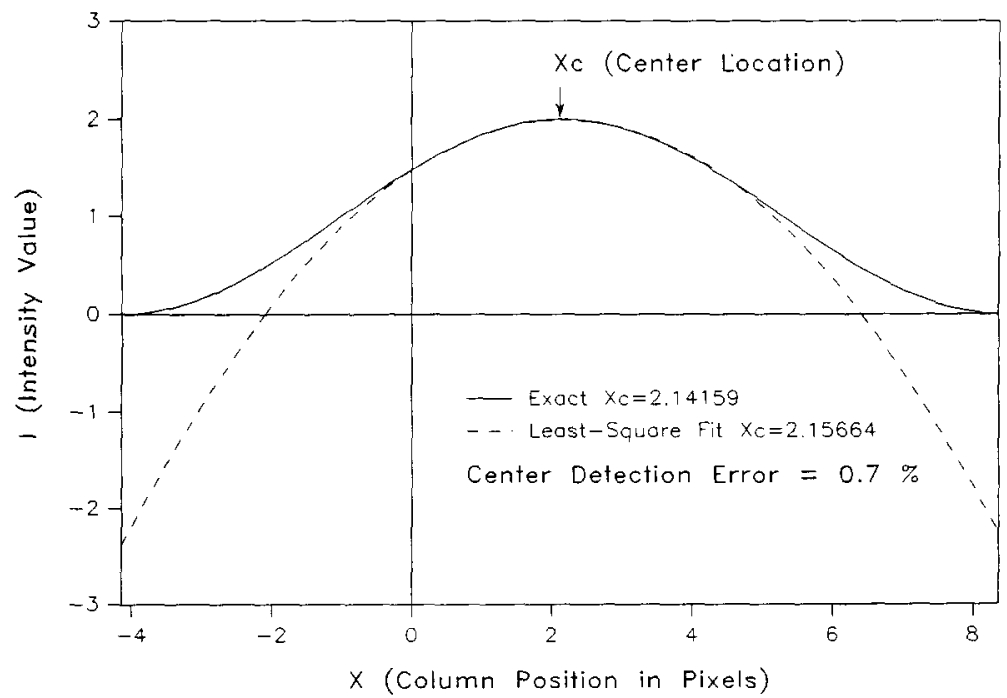

Fig. 2. Intensity distribution function $I=A \sin (a x+b)+B(A=1, B=1, a=0 \cdot 5$, $b=0 \cdot 5)$. 


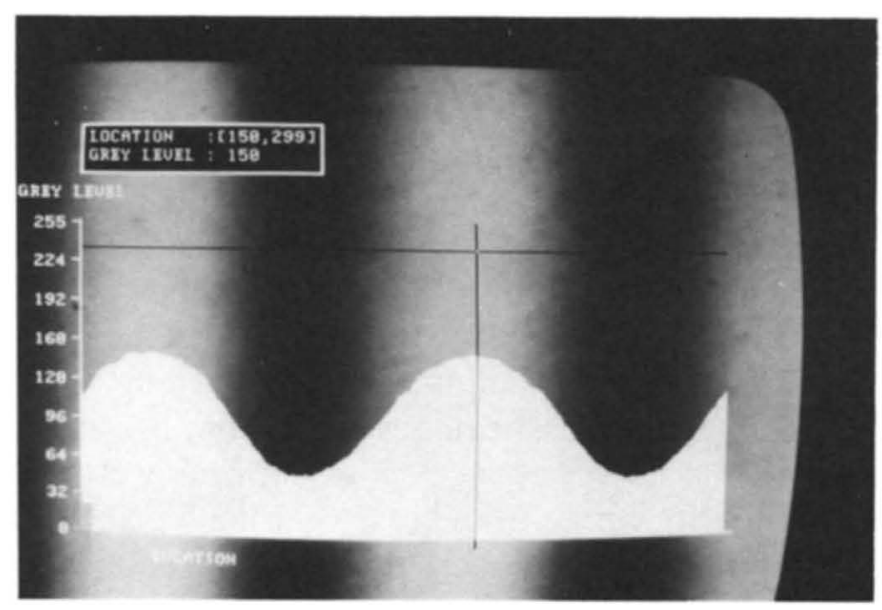

Fig. 3. Sinusoidal distribution between two adjacent fringes.

the comparison between the exact and the estimated centers. The theoretical error of fringe center detection is estimated about $0.7 \%$ if 5-pixel data were used. It should be noted that the error is based on the following assumptions:

(1) the width of the scanned fringe is about 5 pixels;

(2) five pixel points are used in the curve fitting, and two of the five points $\left(x_{-1}\right.$ and $\left.x_{0}\right)$ are exactly $0 \cdot 5$ pixels away from the exact center, $x=2 \cdot 14159$, which represents the worst case;

(3) no random noise is considered.

If the coordinate origin is shifted to $x_{0}$ (near the exact center $x_{\mathrm{c}}$ ), the values of $\alpha x$ will be much smaller than unity. Thus, $\sin (\alpha x) \approx \alpha x$ and $\cos (\alpha x) \approx 1-\alpha^{2} x^{2} / 2$, so that

$$
I(x) \approx a_{2} x^{2}+a_{1} x+a_{0}
$$

where $a_{2}=-\left(A \alpha^{2} \sin \beta\right) / 2, \quad a_{1}=A \alpha \cos \beta$, and $a_{0}=B+A \sin \beta$. In fact, eqn (4) can be derived from eqn (3) when $x_{\mathrm{c}}$ is set to zero, which corresponds to $\beta=\pi / 2$. To be computationally efficient, eqn (4) will be used throughout this paper to approximate the sinusoidal function described in eqn (2). Figure 3 shows an example of the fringe center detected from a sinusoidal intensity distribution between two adjacent fringes.

\section{Optimum reconstruction filter}

As can be seen in Fig. 3, only a small central portion of the sinusoidal curve can be approximated by a polynomial. The accuracy of fringe 
center detection relies on the number of pixels taken and the type of curve fitting used to reconstruct the intensity curve. An optimum reconstruction filter can then be developed based on the relationship between the fringe width and the number of pixels taken, and the type of curve fitting used. Tables 1 and 2 show the error comparisons of computer simulation in detecting the fringe centers along a theoretical circular arc. Detected centers are compared with the theoretical fringe center location in the same row (i.e. the same horizontal scanned line). Several types of curve fitting methods such as the Lagrange interpolation, cubic-spline function, least-squares regression, B-spline curves and Bernstein polynomial curves using 3,5 or 7 pixels were employed to reconstruct an intensity curve. In the case of using 5 pixels, for instance, the approximately detected center position is designated as position 0 , and 2 pixels before and after the position 0 are designated as positions $-2,-1,1$ and 2 , respectively. The estimated fringe center is located at the maximum or minimum of $I(x)$. This can be easily found by taking the first derivative of the $I(x)$ and using the bisection method to find the root of $I^{\prime}(x)=0$. The bisection method guarantees to find the root within a specified interval (for instance, the selected 5 pixels). It should be noted that the bright and dark fringes are assumed equal in width. Thus, the wavelength indicated in Tables 1 and 2 is essentially the sum of bright and dark fringes.

It is more meaningful to compare the detection errors shown in Table 2 when random noise effect is considered. It can be seen that the errors based on 1-pixel resolution are generally 10-20 times as large as those based on sub-pixel resolution. Among various curve fitting methods presented, the Lagrange interpolation and least-squares regression produce better results. It is found that the errors mainly depend upon the wavelength and the number of pixels used for curve fitting.

The optimum reconstruction filter is described as follows:

(1) the optimum number of consecutive pixels to be used for curve fitting is five;

(2) the optimum curve fitting method is least-squares regression if the wavelength is approximately 14 pixels; or the Lagrange interpolation if the wavelength is approximately 9 pixels. It should be noted that the wavelength consists of bright and dark fringes of equal width.

As indicated in Tables 1 and 2, the optimum reconstruction filter based on sub-pixel resolution exhibits high accuracy in fringe center detection and low sensitivity to noise. Depending upon the applications, sometimes the wavelength has to be relatively small (e.g. 9 pixels) to 


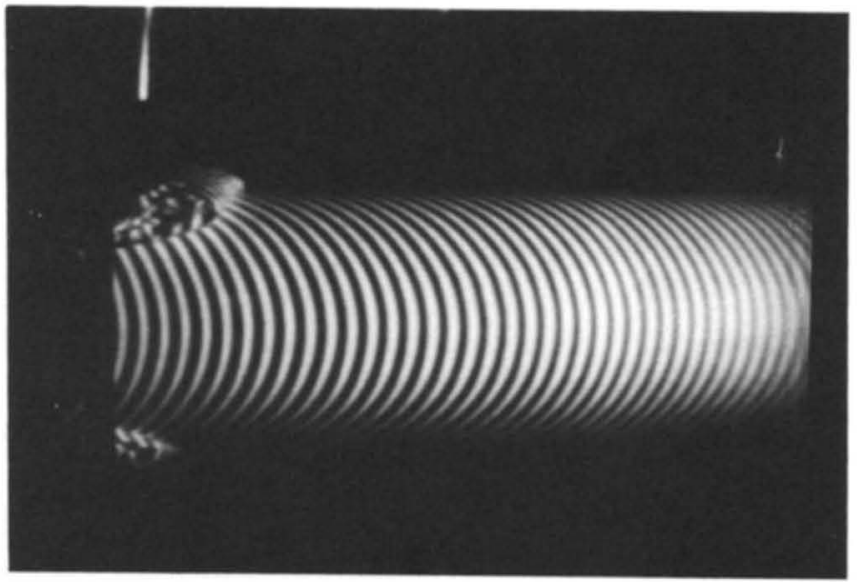

Fig. 4. Projected fringes on a cylinder.

generate more data points in an acquired image. In such a case, Lagrange interpolation is the recommended curve fitting method.

\section{APPLICATION 1: RADIUS MEASUREMENT OF CYLINDRICAL OBJECTS}

The first application of fringe center detection technique is radius measurement of cylindrical objects. As shown in Fig. 4, a series of elliptical fringes are generated by projecting a sinusoidal grating onto a cylinder. Theoretically, it is possible to fit a general second-order curve of the following form:

$$
A X^{2}+B X Y+C Y^{2}+D X+E Y+F=0
$$

Orthographic projection is used in experiments, which means that the camera (image) plane is parallel to the major axis of the cylinder. When the angle $\theta$ between the incident light and the cylinder axis is less than $45^{\circ}$, the minor axis of the ellipse is along the vertical axis of the image coordinate, and its length is equal to the diameter of the cylinder. As $\theta$ becomes larger than $45^{\circ}$, the major axis of the ellipse is along the vertical axis, and its length is equal to the diameter of the cylinder. This implies that the image of the ellipse is a circle when the angle is exactly $45^{\circ}$, and the diameter of the circle is essentially the diameter of the cylinder.

Dividing eqn (5) by $F$ gives

$$
a x^{2}+b x y+c y^{2}+d x+e y+1=0
$$


Mathematically, only five arbitrary data points (fringe centers) along a fringe are needed to determine the five coefficients from which the lengths of both major and minor axes of a ellipse can be calculated. However, it is inaccurate to rely on only five points, as the coefficients of the second-order equation are highly coupled and sensitive to measurement noise. To be more accurate, we can use many data points to determine the coefficients using a least-sqares fitting technique. The most accurate and reliable method is circle fitting when the angle $\theta$ is exactly $45^{\circ}$. In theory, only three points are needed to determine a circle. Nevertheless, owing to measuring errors, it is desirable to use as many points along the circular arc as possible. The radius of a circle can be calculated through least-squares circle fitting. The calculation is shown as follows:

$$
\begin{aligned}
x_{\mathrm{c}} & =-A / 2 \\
y_{\mathrm{c}} & =-B / 2 \\
r & =\left(x_{\mathrm{c}}^{2}+y_{\mathrm{c}}^{2}-c\right)^{1 / 2}
\end{aligned}
$$

where $\left(x_{\mathrm{c}}, y_{\mathrm{c}}\right)$ and $r$ are the center and radius of the fitted circle, respectively. The radius $r$ can be determined by solving the coefficients $A, B$ and $C$ :

$$
\left[\begin{array}{ccc}
\Sigma\left(x^{2}\right) & \sum(x y) & \sum(x) \\
\sum(x y) & \sum\left(y^{2}\right) & \sum(y) \\
\Sigma(x) & \Sigma(y) & N
\end{array}\right]\left[\begin{array}{l}
A \\
B \\
C
\end{array}\right]=\left[\begin{array}{c}
-\sum\left(x^{3}\right)-\sum\left(y^{2} x\right) \\
-\sum\left(y x^{2}\right)-\sum\left(y^{3}\right) \\
-\sum\left(x^{2}\right)-\sum\left(y^{2}\right)
\end{array}\right]
$$

where $x$ and $y$ are the locations of fringe centers, and $N$ is the total number of points. The computer simulation and experimental results

\begin{tabular}{|c|c|c|c|c|}
\hline & \multicolumn{2}{|c|}{$\begin{array}{c}\text { Computer simulation } \\
\text { results }\end{array}$} & \multicolumn{2}{|c|}{$\begin{array}{l}\text { Experimental } \\
\quad \text { results }\end{array}$} \\
\hline & $\begin{array}{l}\text { One-pixel } \\
\text { resolution }\end{array}$ & $\begin{array}{l}\text { Sub-pixel } \\
\text { resolution }\end{array}$ & $\begin{array}{l}\text { One-pixel } \\
\text { resolution }\end{array}$ & $\begin{array}{l}\text { Sub-pixel } \\
\text { resolution }\end{array}$ \\
\hline $\begin{array}{l}\text { Actual } \\
\text { radius }\end{array}$ & 116 & 116 & 116 & 116 \\
\hline $\begin{array}{l}\text { Calculated } \\
\text { radius }\end{array}$ & $116 \cdot 3976$ & $116 \cdot 0342$ & $116 \cdot 6186$ & $115 \cdot 9416$ \\
\hline Error $(\%)$ & $0 \cdot 3428$ & 0.0295 & 0.5333 & 0.0503 \\
\hline
\end{tabular}
thus obtained using eqn (8) are shown in Table 3.

TABLE 3

Comparison of Accuracy (Values Given in Pixels) in Cylinder Radius Measurement 


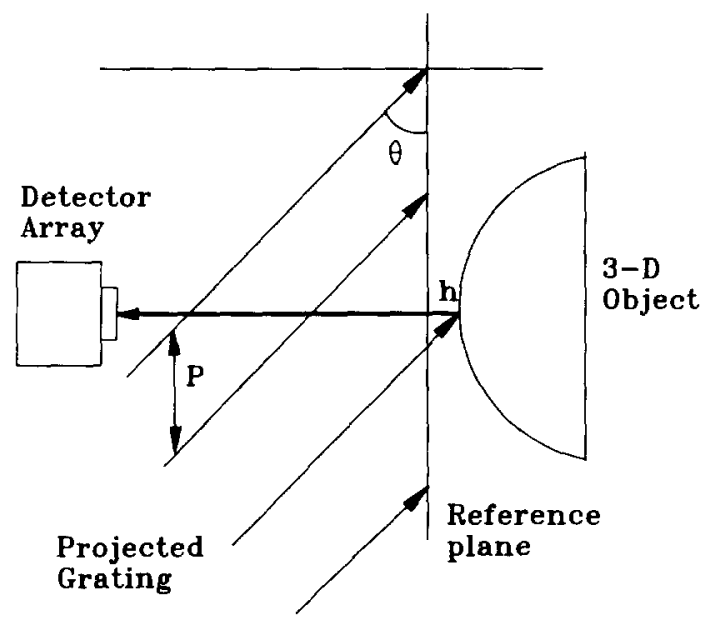

Fig. 5. Geometry of surface profile measurement.

\section{APPLICATION 2: SURFACE PROFILE MEASUREMENT OF DIFFUSE OBJECTS}

In the second application, surface measurement of diffuse objects is presented. As shown in Fig. 5, when a sinusoidal grating is projected onto a three-dimensional diffuse object, the object surface height distribution is translated to a phase distribution of the deformed fringes. The height can be determined ${ }^{6}$ by

$$
h(x, y)=(p \tan \theta / 2 \pi) \phi(x, y)
$$

where $h(x, y)$ is the object height at any point $(x, y)$ relative to an arbitrarily chosen reference plane which is parallel to the detector array, $p$ is the grating pitch in the reference plane, $\theta$ is the grating projection angle inclined to the reference plane, and $\phi(x, y)$ is the phase distribution of the deformed fringes. When the phase values on each fringe center are detected and translated into surface profile, the height at the center of each bright fringe is given by

$$
h=n p \tan \theta, \quad n=0, \pm 1, \pm 2, \ldots
$$

Likewise, the height at the center of each dark fringe is

$$
h=(n+1 / 2) p \tan \theta, \quad n=0, \pm 1, \pm 2, \ldots
$$

Recently, Chang and $W^{6}{ }^{6}$ proposed a normalization process in their simple automatic phase reduction algorithm. Through this process, not only can the intensity amplitude be more uniform, which is important if 
the phase reduction error is to be reduced, but also the fixed pattern noise can be subtracted. The major advantage of their algorithm is that the necessity of using a vibration-free table is eliminated. The work presented in this paper used this algorithm to reduce the phase reduction error. As the deformed fringes result from the interference of a tilt wavefront whose phase increases monotonically with respect to the reference wavefront, the surface can be reconstructed by accurately locating a series of fringe centers, translating them into a height distribution, interpolating the surface points, and finally removing the tilt.

The accuracy of surface reconstruction relies on the accuracy of fringe center detection with sub-pixel resolution. The object to be profiled here is a free curve surface as shown in Fig. 6(a). Figure 6(b) shows the reconstructed three-dimensional surface profile as a result of using eqns (10) and (11) based on sub-pixel resolution. To verify the experimental result, the measured height data are compared with those obtained from a coordinate measuring machine (CMM) with $1-\mu \mathrm{m}$ resolution. Figure $6(\mathrm{c})$ gives the comparison of the measured data for a cross-section in the central region. Using the CMM data as reference, the use of sub-pixel resolution results in a maximum error of less than $0.1 \mathrm{~mm}$ and average error of about $30 \mu \mathrm{m}$. As can be seen in eqn (9), the measurement sensitivity is mainly dependent on the choice of effective wavelength $p \tan \theta$. A smaller system wavelength will generate more data points, which should result in better accuracy in 3D surface profilometry.

\section{CONCLUSIONS}

An accurate fringe detection technique based on sub-pixel resolution is presented. The developed optimum reconstruction filter has low sensitivity to noise. Computer simulation and experimental results indicate that the geometry measurements become much more accurate and precise using the presented sub-pixelation technique. In terms of fringe center detection, the detecting error ratio between using the proposed sub-pixel resolution and the conventional 1-pixel resolution (as shown in Table 1) is generally less than $0 \cdot 1$. In terms of applications, the measuring error ratio is about 0.1 for cylinder radius measurement and 0.4 for surface profile measurement.

The high accuracy is achieved by the employment of sub-pixel resolution, and the high repeatability is achieved through an optimum reconstruction filter. In referring to measurements, the terms 'accuracy' 


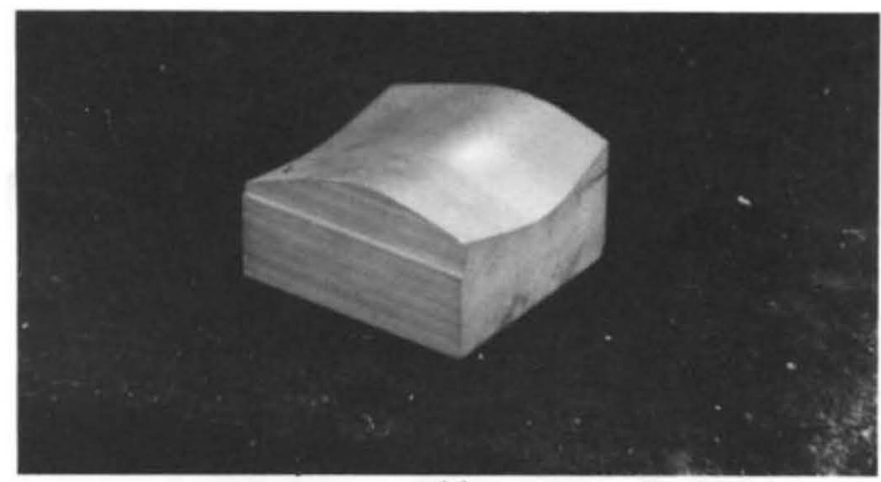

(a)

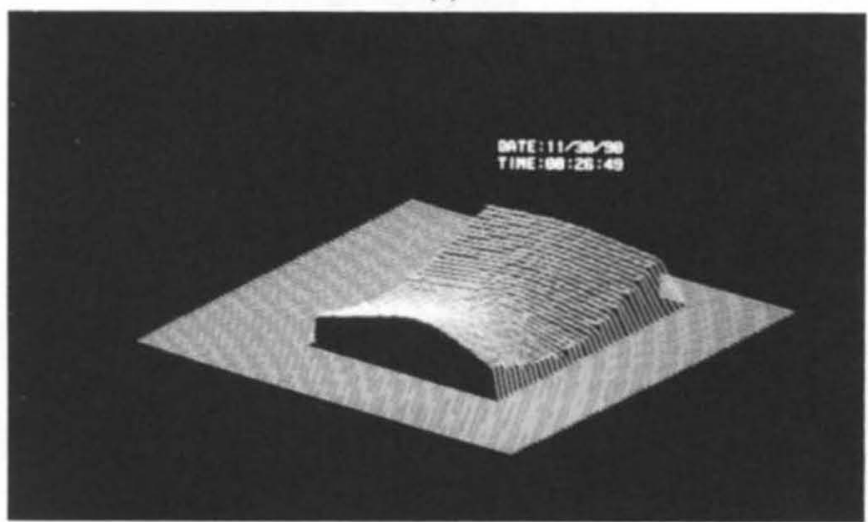

(b)

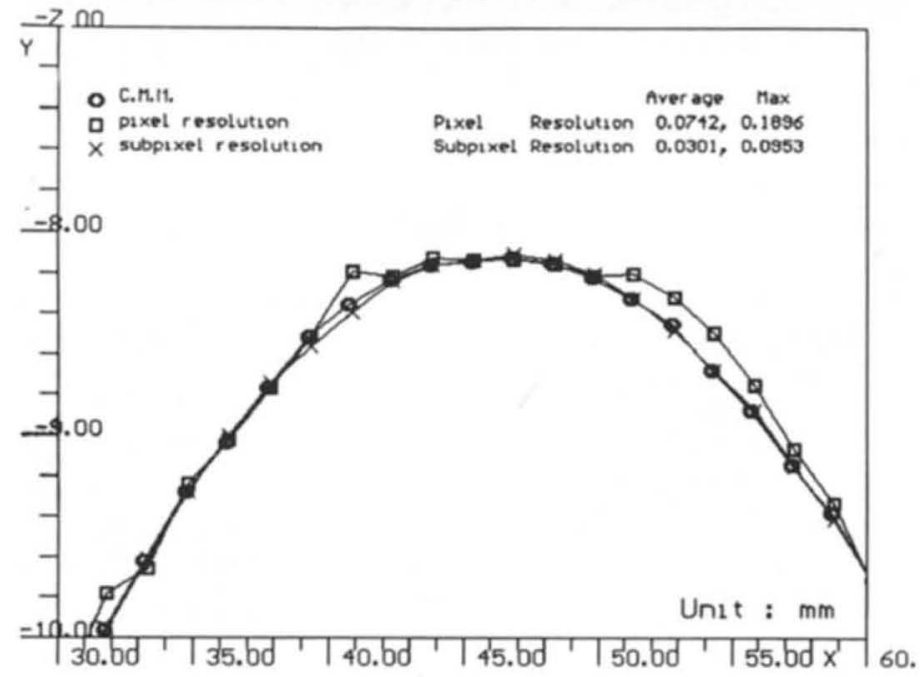

(c)

Fig. 6. (a) Free curve surface model; (b) reconstructed 3D surface; (c) comparison of measured height data (values in millimeters). 
and 'precision' have different meanings: accuracy is understood to indicate how closely a measurement relates to an agreed upon standard or truth, and the precision of a measurement is referred to as a degree of repeatability. ${ }^{7}$ It is possible to have precision without accuracy, but it is not possible to have repeatable accuracy without precision. Once the precision is obtained, accuracy can be calibrated into the instrument. From this study, it can be concluded that the presented sub-pixelation must be accompanied by an optimum reconstruction filter.

\section{ACKNOWLEDGEMENTS}

The authors acknowledge the support of the National Science Council of Taiwan, under project number NSC81-0422-E033-01, and the use of computing facilities at the Cleveland State University, Ohio, USA.

\section{REFERENCES}

1. Takasaki, H., Moiré topography. Appl. Opt., 9 (1970), 1457-72.

2. Idesawa, M. \& Yatagai, T., General theory of projection-type moiré topography. Sci. Papers IPCR, 71 (1979), 57-70.

3. Srinivasan, V., Liu, H. C. \& Halioua, M., Automated phase-measuring profilometry of 3D diffuse objects. Appl. Opt., 23 (1984) 3105-8.

4. Lin, P. P., Parvin, F. \& Shoenig, F., Jr, Optical gaging of very-short term surface waviness. Trans. North Am. Manuf. Res. Inst. Society of Manufacturing Engineers (SME), Dearborn, Michigan, 1991, 327-32.

5. Wan, D. S. \& Lin, D. T., Ronchi test and a new phase reduction algorithm. Appl. Opt., 29 (1990), 3255-65.

6. Chang, M. \& Wan, D. S., On-line automated phase-measuring profilometry. Optics Lasers Eng., 15 (1991), 127-39.

7. South, V., III, Automatic precision video measurement for quality inspection. In Gaging with Vision Systems. Society of Manufacturing Engineers (SME), Dearborn, Michigan, 1987, pp. 70-8. 